\title{
A design of planar multi-turn flux transformers for radio frequency SQUID magnetometers
}

Y. Zhang, H. R. Yi, J. Schubert, W. Zander, M. Banzet, and A. I. Braginski

Citation: Appl. Phys. Lett. 72, 2029 (1998);

View online: https://doi.org/10.1063/1.121254

View Table of Contents: http://aip.scitation.org/toc/apl/72/16

Published by the American Institute of Physics

\section{Articles you may be interested in}

Superconducting multiturn flux transformers for radio frequency superconducting quantum interference devices Journal of Applied Physics 88, 5966 (2000); 10.1063/1.1322382

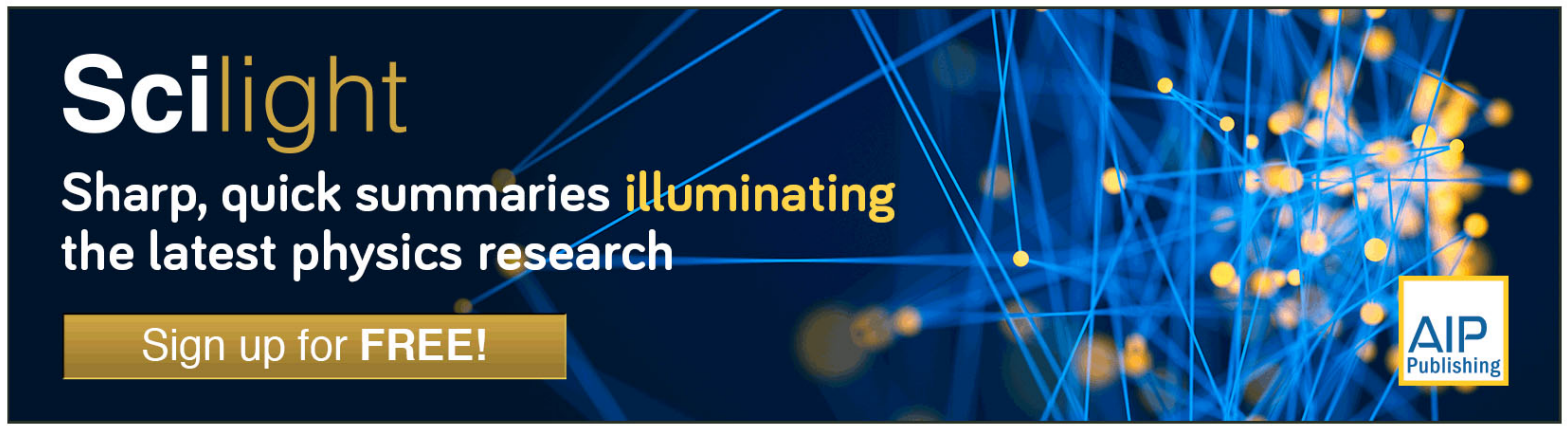




\title{
A design of planar multi-turn flux transformers for radio frequency SQUID magnetometers
}

\author{
Y. Zhang, H. R. Yi, ${ }^{\text {a) }}$ J. Schubert, W. Zander, M. Banzet, and A. I. Braginski \\ Institut für Schicht-und Ionentechnik (ISI), Forschungszentrum Jülich (FZJ) GmbH, D-52425 Jülich, \\ Germany
}

(Received 5 December 1997; accepted for publication 18 February 1998)

\begin{abstract}
We propose a design for planar multi-turn flux transformer for radio frequency (rf) superconducting quantum interference device (SQUID) magnetometers. This transformer is integrated with a coplanar resonator, e.g., on a $1 \mathrm{~cm}^{2} \mathrm{LaAlO}_{3}$ substrate. Its pickup loop is connected with two input coils, separate for dc (low-frequency) and rf currents. A double-hole washer SQUID is coupled to these coils in a flip-chip configuration to form a magnetometer. The separation of $\mathrm{rf}$ and dc current paths in the transformer made it possible to demonstrate the rf SQUID magnetometer operation.

(C) 1998 American Institute of Physics. [S0003-6951(98)01916-0]
\end{abstract}

Planar multi-turn flux transformers have been widely used to increase the field sensitivity of high temperature superconductor (HTSs) dc superconducting quantum interference device (SQUID) magnetometers. ${ }^{1,2}$ However, no work has yet been reported on the multi-turn flux transformer coupled to a HTS radio frequency (rf) SQUID. The superconducting coplanar resonator surrounding a flux concentrator coupled to a small rf washer SQUID in a flip-chip configuration $^{3,4}$ provides the possibility of using a similar design of multi-turn flux transformer as in dc SQUID technology. We hoped to simply replace the flux concentrator (single-turn transformer) shown in Fig. 1(a) with a multi-turn flux transformer shown in Fig. 1(b). However, in our experiments, the resonance of the resonator could no longer be observed, once the connecting strip (crossover) was fabricated to close the loop of the transformer.

The two strip lines of the coplanar resonator and the flux transformer form a high frequency resonant system. For the single-layer flux concentrator (or transformer) [Fig. 1(a)], this resonant system is similar to the symmetric slit coplanar resonator (Fig. 2), ${ }^{5}$ where the single-turn flux transformer can be considered as the third strip line. However, the closed multi-turn input coil in the transformer results in broken symmetry of the third strip line. This may greatly decrease the quality factor $Q$ of the resonator or even cause the disappearance of the resonance.

In this letter, we propose a new design concept, which aims at minimizing the influence of the multi-turn input coil on the rf resonant system. In a separate article, we describe the fabrication and characterization of transformers and flipchip magnetometers based on this concept. ${ }^{6}$ It is shown that with this design a planar rf SQUID magnetometer with a flux transformer is feasible.

The inspiration for our new concept came from the seasoned low temperature superconductor SQUID technology. Three successful designs of rf SQUID magnetometers should be evoked, which have been widely referred to.

Zimmermann et al. ${ }^{7}$ invented the two-hole SQUID [Fig. 3(a)], which consisted of two bulk superconducting rings

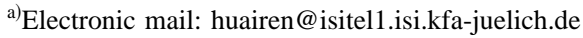

joined by a common Josephson junction. In one hole, a coil of inductance $L$ together with a capacitor $C$ formed a tank circuit having a resonant frequency of about $20 \mathrm{MHz}$. In another hole a superconducting coil $L_{\text {in }}$ was connected to the pickup loop forming the magnetic flux input circuit. Note that a bare two-hole SQUID is a gradiometer. Its sensitivity to a uniform external magnetic field is zero. However, it detects the gradient of the external magnetic field in an unshielded environment.

Kamper and Zimmerman $^{8}$ developed the toroidal SQUID shown in Fig. 3(b). In the bulk niobium structure, a niobium point contact provided a weak link in the center. Two coils were buried in two concentric circle slot. The inner coil $L$ and the capacitor $C$ formed the tank circuit. The outer superconducting coil served as the input coil $L_{\text {in }}$ for the dc signal. There are two advantages to this design. One is the good self-shielding of the SQUID from external magnetic field and gradient. Another is the excellent coupling between the input coil and the SQUID.

The third design was the microwave SQUID [Fig. 3(c)]. ${ }^{9}$ Its pumping frequency was about $10 \mathrm{GHz}$. This design employed a thin film of an InSn alloy deposited on a hollow cylindrical substrate. The film was cut mechanically leaving a Dayem bridge as the sole circumferential current path forming a single-hole rf SQUID. As indicated in Fig. 3(c), a loop surrounding the SQUID was connected to the microwave source and readout electronics through a circulator. The input flux was coupled to the SQUID by means of an internal superconducting input coil.
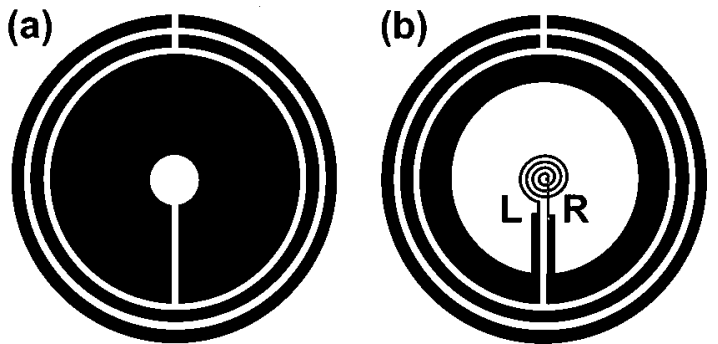

FIG. 1. A coplanar resonator integrated with (a) a single-layer flux concentrator and (b) a multi-turn flux transformer. $L$ and $R$ indicate the two sides of the slit. 


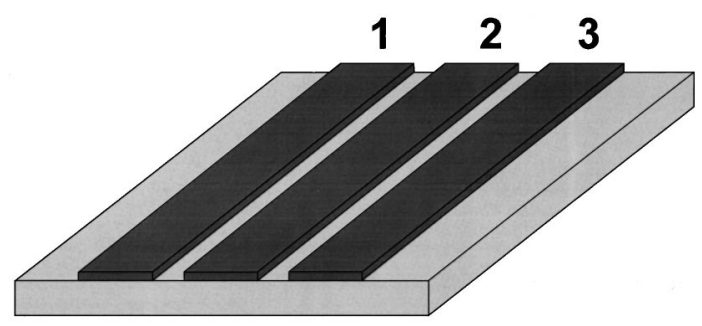

FIG. 2. A standard slit coplanar resonator formed with three strip lines on a dielectric substrate.

A common feature of the three magnetometers was that their tank circuit and the dc flux input coil were separated. Separate coils were used to couple the rf and dc (lowfrequency) currents to the SQUID, respectively. Here, we decided to pursue a similar separation principle. Among the three designs, the two-hole SQUID design can most likely be adapted to a planar layout and integrated with the coplanar resonator in the same substrate.

We already pointed out that the broken symmetry of the third strip line may lead to a sharp drop of $Q$ or even the disappearance of the resonance. Let us introduce a shunt at the two sides ( $L$ and $R$ ) of the slit separating the input leads of the multi-turn input coil of Fig. 1(b). This can be done by depositing a highly conducting thin-film strip of $\mathrm{Au}$ or $\mathrm{Ag}$. Now, high-frequency current will pass largely across the shunt, because of the high impedance of the multi-turn superconducting coil for high frequency currents. As a consequence, the symmetry of the high-frequency system should be improved greatly. However, for the low frequency detection signal $(\mathrm{dc})$, the current should be passing through the

(a)

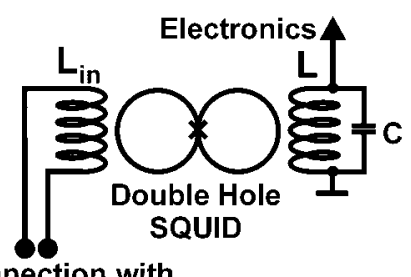

Connection with

Pickup Loop

(b)
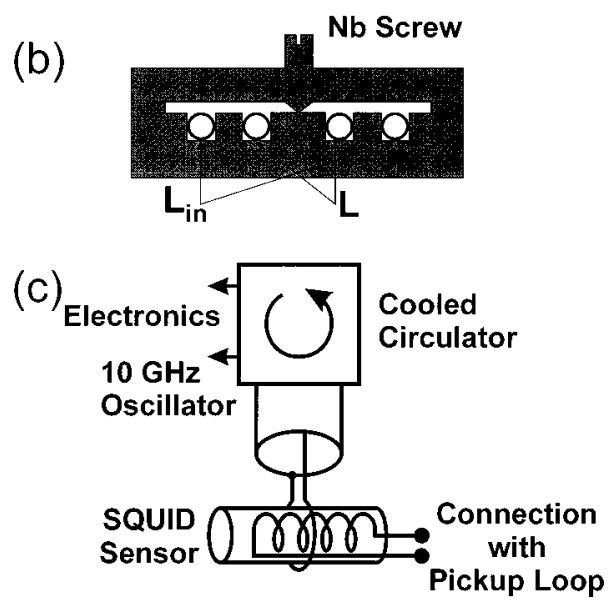

FIG. 3. Three designs of low temperature superconductor rf SQUID magnetometer. (a) Equivalent circuit of the two-hole SQUID magnetometer. (b) Schematic (cross section) of the toroidal SQUID with two coils, $L$ and $L_{\text {in }}$. (c) Configuration of a microwave thin film single-hole SQUID magnetometer. (a)

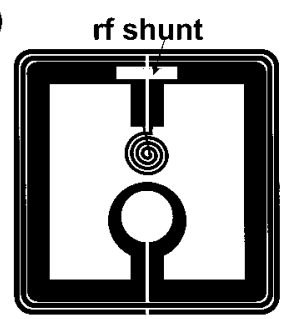

(b)

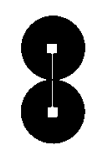

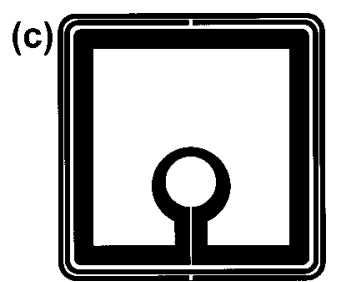

(d)

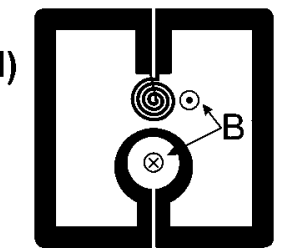

FIG. 4. Layout and equivalent circuits of a new planar rf SQUID magnetometer. (a) A coplanar resonator integrated with a flux transformer with a metal strip shunting the multi-turn input coil. (b) A double-hole washer SQUID. (c) Equivalent circuit for rf currents. (d) Equivalent circuit for dc currents.

superconducting multi-turn input coil. The metal shunt will have no effect on the dc supercurrent.

The reasoning presented above was confirmed experimentally. Once the shunt was introduced, the coplanar resonator resonated again. A washer SQUID was then coupled to the center of the input coil by a flip-chip arrangement. However, no SQUID signals could be observed. This is an indication that, due to the metal shunt, the rf signal indeed did not pass through the multi-turn input coil, i.e., the coupling between the resonator and the SQUID was very weak. Obviously, the design in Fig. 1(b) cannot be used for a planar rf SQUID magnetometer with coplanar resonator.

The new, more practical planar layout concept is shown in Fig. 4(a). The two strip lines of the coplanar resonator surround the flux transformer, which includes two separate input coils and a common pickup loop inductively coupled to the resonator. The single-turn rf coil and the dc multi-turn coil are wound in opposite directions. The multi-turn input coil is shunted by a normal-conducting strip. A planar double-hole washer SQUID shown in Fig. 4(b) is placed on top of the structure in the flip-chip configuration such that the SQUID holes are coupled to the two input coils.

This layout has two equivalent circuits. For rf current, because of the metal shunt, this flux transformer can be viewed as a single-turn flux transformer of Fig. 4(c). It has been proven that this single-turn flux transformer has a good high-frequency coupling with the washer rf SQUID. ${ }^{4}$ The dc circuit is shown in Fig. 4(d). The supercurrent induced by external dc flux threading the pickup coil produces two opposite magnetic fields of the two input coils in two holes of the SQUID. The resulting SQUID currents are added in the common junction so that a planar magnetometer is obtained. It should be noted that the planar single-turn input coil not only couples rf current but also dc current. Hence, this design should be somewhat better than the bulk double-hole SQUID. The appropriate inductance of the double-hole washer SQUID should be in the range of 200-250 pH, since the best magnetic field sensitivity was obtained for singlehole washer SQUIDs with these inductance values. ${ }^{4}$

Flux transformers based on the presented concept were fabricated on $\mathrm{LaAlO}_{3}$ substrates and with $\mathrm{LaAlO}_{3}$ insulation as presented elsewhere. ${ }^{6}$ The pickup loop had an outer di- 


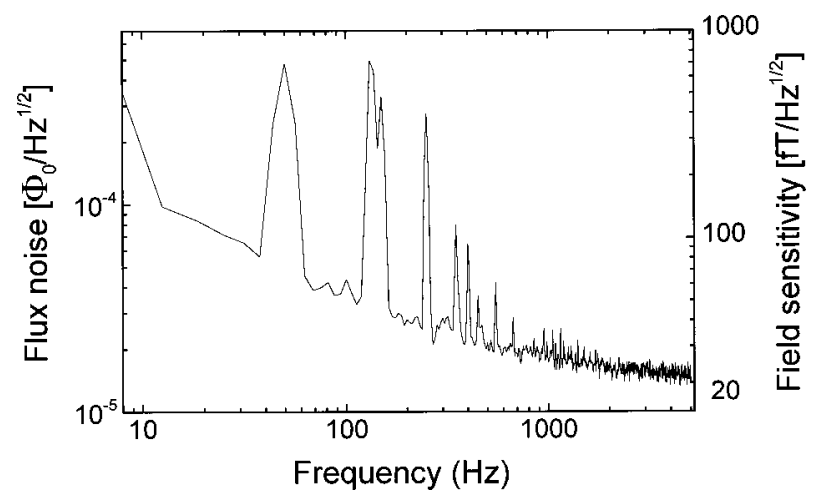

FIG. 5. Noise spectrum of a magnetometer consisting of a transformer coupled to a double-hole washer SQUID with two equal $30 \times 750 \mu \mathrm{m}^{2}$ loops.

mension of $8 \times 8 \mathrm{~mm}^{2}$ and a linewidth of $0.8 \mathrm{~mm}$. The input coil had nine turns with both the linewidth and separation between turns of $20 \mu \mathrm{m}$. The double-hole SQUID with a step-edge junction had two equal $30 \times 750 \mu \mathrm{m}^{2}$ loops with washers $1.5 \mathrm{~mm}$ in diameter. The total SQUID inductance was $L_{s} \approx 230 \mathrm{pH}$. Several transformers of this design were measured with the same flip-chip SQUID. The fully characterized set had a tank frequency of $840 \mathrm{MHz}$, the unloaded $Q_{0}=600$, and the measured flux-to-field coefficient of 1.45 $n T / \Phi_{0}$. At $5 \mathrm{kHz}$ signal frequency, the white noise measured in magnetic shielding was $1.5 \times 10^{-5} \Phi_{0} / \mathrm{Hz}^{1 / 2}$ corresponding to a field resolution of $22 \mathrm{fT} / \mathrm{Hz}^{1 / 2}$. At $100 \mathrm{~Hz}$, a field resolution of $50 \mathrm{fT} / \mathrm{Hz}^{1 / 2}$ was obtained. The measured noise spectrum is shown in Fig. 5.

In conclusion, the feasibility of the presented design concept was unambiguously confirmed. The optimization of the designs and of the multilayer technology, as well as the reduction of the low-frequency $1 / f$ type noise, are the tasks of the future. In closing, let us emphasize that for dc SQUID there is only one coupling issue, i.e., the coupling of the external flux to the SQUID. For the optimized operation, one needs only to match inductances of the pickup loop and the input coil while keeping a high mutual inductance between the input coil and the SQUID. However, in the case of rf SQUID one must provide both the dc and the rf coupling, with the latter satisfying $k^{2} Q>1$, where $k$ is the rf coupling coefficient. We could show that the separation of the rf and $\mathrm{dc}$ current paths provides a satisfactory solution to the problem.

This work was supported by the German BMBF under Contract No. 13 N 6682.

${ }^{1}$ D. Drung, F. Ludwig, W. Müller, U. Steinhoff, L. Trahms, H. Koch, Y. Q. Shen, M. B. Jensen, P. Vase, T. Holst, T. Freltoft, and G. Curio, Appl. Phys. Lett. 68, 1421 (1996).

${ }^{2}$ D. Reimer, M. Schilling, S. Knappe, and D. Drung, IEEE Trans. Appl. Supercond. 5, 2342 (1995).

${ }^{3}$ Y. Zhang, W. Zander, J. Schubert, F. Rüders, H. Soltner, M. Banzet, N. Wolters, X. H. Zeng, and A. I. Braginski, Appl. Phys. Lett. 71, 704 (1997).

${ }^{4}$ Y. Zhang, N. Wolters, X. H. Zeng, J. Schubert, W. Zander, H. Soltner, H. R. Yi, M. Banzet, F. Rüders, and A. I. Braginski, Appl. Supercond. (submitted).

${ }^{5}$ J. B. Knorr and K. D. Kuchler, IEEE Trans. Microwave Theory Tech. 23, 541 (1975).

${ }^{6}$ H. R. Yi, J. Schubert, W. Zander, X. H. Zeng, M. Banzet, D. F. He, and Y. Zhang, Supercond. Sci. Technol. (submitted).

${ }^{7}$ J. E. Zimmerman, P. Thiene, and J. T. Harding, J. Appl. Phys. 41, 1572 (1970).

${ }^{8}$ R. A. Kamper and J. E. Zimmerman, J. Appl. Phys. 42, 132 (1971).

${ }^{9}$ J. M. Pierce, J. E. Opfer, and L. H. Rorden, IEEE Trans. Magn. MAG 10, 599 (1974). 